PROCEEDINGS OF THE

AMERICAN MATHEMATICAL SOCIETY

Volume 102, Number 1, January 1988

\title{
A GENERALIZED CAPACITY AND A UNIQUENESS THEOREM ON THE DYADIC GROUP
}

\author{
KAORU YONEDA
}

(Communicated by Richard R. Goldberg)

\begin{abstract}
In this paper we shall introduce a generalized capacity and give a necessary and sufficient condition for a subset of the dyadic group to be a $U$-set for a certain class of Walsh series.
\end{abstract}

Wade [2] proved that a a subset of the dyadic group is a $U$-set for $\tau_{\alpha}^{+}$if and only if it is of $\alpha$-capacity zero. We have already partially generalized Wade's theorem in [4].

A dyadic interval of rank $n, I_{n}^{p}$, is the set of all 0-1 series, $x=\left(t_{1}, t_{2}, \ldots\right)$, such that $\sum_{k=1}^{n} t_{k} / 2^{k}=p / 2^{n}$. $I_{n}(x)$ is the dyadic interval of rank $n$ which contains $x$. Hence $I_{0}^{0}$ is the dyadic group.

For convenience we shall write $x=\left(\sum_{k=1}^{\infty} t_{k} / 2^{k}\right)^{-}$if $\lim _{k \rightarrow \infty} t_{k}=1$ and $x=$ $\left(\sum_{k=1}^{\infty} t_{k} / 2^{k}\right)$ otherwise. We refer the reader to [3 and 4] for details of the dyadic group.

A dyadic measure $m$ is a real valued set function satisfying the additive law $m\left(I_{n}^{p}\right)=m\left(I_{n+1}^{2 p}\right)+m\left(I_{n+1}^{2 p+1}\right)$ for $n=0,1, \ldots, p=0,1, \ldots, 2^{n}-1$. When $\mu \equiv$ $\sum_{k=0}^{\infty} \hat{\mu}(k) w_{k}(x)$ is an arbitrary Walsh series, set

$$
m_{\mu}\left(I_{n}^{p}\right)=\lim _{N \rightarrow \infty} \int_{I_{n}^{p}} \sum_{k=0}^{N} \hat{\mu}(k) w_{k}(x) d x=1 / 2^{n} \sum_{k=0}^{2_{n}-1} \hat{\mu}(k) w_{k}\left(p / 2^{n}\right)
$$

for each dyadic interval $I_{n}^{p}$. It is easy to see that $m_{\mu}$ is a dyadic measure and

$$
\hat{\mu}(k)=\int_{I_{0}^{0}} w_{k}(x) m_{\mu}(d x) \equiv \int_{0}^{1^{-}} w_{k}(x) m_{\mu}(d x) \text { for all } k .
$$

Conversely an arbitrary dyadic measure always has its Walsh Fourier expansion [3].

Let $\varepsilon=\left\{\varepsilon_{n}\right\}_{n}$ be a sequence of positive numbers such that

$$
1>\varepsilon_{0}>\varepsilon_{1}>\cdots>\varepsilon_{n}>\cdots>0 \text { and } \lim _{n \rightarrow \infty} \varepsilon_{n}=0 .
$$

Since $\sum_{k=2^{n}}^{2^{n+1}-1} w_{k}(x)=2^{n} w_{2^{n}}(x)$ for $x \in I_{n}^{0}(n=0,1, \ldots)$ and $=0$ otherwise,

$$
\alpha(x)=1+\sum_{n=0}^{\infty} \varepsilon_{n} \sum_{k=2^{n}}^{2^{n+1}-1} w_{k}(x)
$$

Received by the editors September 17, 1986.

1980 Mathematics Subject Classification (1985 Revision). Primary 42C25, 28 A12.

Key words and phrases. Walsh function, set of uniqueness, $\alpha$-capacity. 
converges uniformly on each $I_{0}^{0} \backslash I_{n}^{0}$ and $\alpha(x)$ is a positive and integrable function. It is obvious that

$$
\int_{0}^{1^{-}} \alpha(x) d x=1, \quad \alpha(x)=\text { constant on } I_{n}^{1}, \quad n=1,2, \ldots, \alpha(x) \geq \alpha(y),
$$

if $x<y, \alpha(x)=\sum_{n=0}^{\infty} a_{n} \chi_{I_{n}^{0}}(x)$, where $\chi_{I}$ is the characteristic function of $I$, and $\sum_{n=0}^{\infty} a_{n} / 2^{n}=1$.

For each Borel set $E \subseteq I_{0}^{0}, \mathfrak{M}(E)$ denotes the set of all Walsh Fourier series of nonnegative dyadic measures concentrated with $E$ with total variation 1. For each $\mu \in \mathfrak{M}(E)$, the potential function of $\mu, U_{\mu}$, and the energy of $\mu, I_{\mu}$, are defined as follows:

$$
\begin{aligned}
& U_{\mu}(x)=\int_{0}^{1^{-}} \alpha(x+t) m_{\mu}(d t)=\sum_{n=0}^{\infty} a_{n} m_{\mu}\left(I_{n}(x)\right) \\
& I_{\mu}=\int_{0}^{1^{-}} U_{\mu}(x) m_{\mu}(d x)=\sum_{n=0}^{\infty} a_{n} \sum_{p=0}^{2^{n}-1}\left|m_{\mu}\left(I_{n}^{p}\right)\right|^{2} \\
& \quad=1+\sum_{n=0}^{\infty} \varepsilon_{n} \sum_{k=2^{n}}^{2^{n+1}-1}|\hat{\mu}(k)|^{2} .
\end{aligned}
$$

$C(E)=1 / \inf _{\mu \in \mathfrak{N}(E)}\left\|U_{\mu}\right\|_{\infty}$ is called the $\varepsilon$-capacity of $E$. For a closed set $E$ and $x \notin E$, there exists a dyadic interval $I_{N}(x)$ such that $I_{N}(x) \cap E=\varnothing$ and $m_{\mu}\left(I_{n}^{p}\right)=0$ for $I_{n}^{p} \subseteq I_{N}(x)$. Hence we have

$$
U_{\mu}(x)=\sum_{n=0}^{N-1} a_{n} m_{\mu}\left(I_{n}(x)\right)<\infty
$$

If $U_{\mu}(x)=\infty$, then $x \in E$. If there exists $x_{0}$ such that $U_{\mu}\left(x_{0}\right)=\infty$, then $\left\|U_{\mu}\right\|_{\infty}=\infty$.

We shall state some fundamental lemmas without proofs. The proofs of these lemmas are quite similar to those of theorems stated in the book of Kahane and Salem [1, pp. 31-37].

LEMMA 1. For a closed subset of the dyadic group $E$ we have

(i) $C(E)>0$ if and only if there exists a Walsh series $\mu \in \mathfrak{M}(E)$ such that $I_{\mu}<\infty$ and

(ii) $1 / C(E)=\inf _{\mu \in \mathfrak{M}(E)} I_{\mu} \equiv I(E)$.

LEMMA 2. If $I(E)<\infty$, there exists uniquely a dyadic measure $m_{\mu^{*}}$, which is called the equilibrium dyadic measure of $E$, such that $\mu^{*} \in \mathfrak{M}(E)$ and $I_{\mu^{*}}=I(E)$.

LEMMA 3. If a nonnegative dyadic measure $m_{v}$ satisfies $I_{v}<\infty$, then, for a closed subset $E$, we have

$$
\begin{aligned}
m_{v}(x & \left.\in E: U_{\mu^{*}}(x)<I(E)\right) \\
& \equiv \lim _{n \rightarrow \infty} m_{v}\left(\bigcup I_{n}(x): x \in E, U_{\mu^{*}}(x)<I(E)\right)=0
\end{aligned}
$$


Let $U_{\varepsilon}^{+}$be the set of all Walsh series of nonnegative dyadic measure $\mu$ satisfying

$$
\sum_{n=0}^{\infty} \varepsilon_{n} \sum_{k=2^{n}}^{2^{n+1}-1}\left|\hat{\mu}^{*}(k)\right|^{2}<\infty
$$

where $\varepsilon=\left\{\varepsilon_{n}\right\}_{n}$ satisfies (1). When $\varepsilon_{n}=1 / 2^{n(1-\alpha)}, n=0,1, \ldots, 0<\alpha<1, U_{\varepsilon}^{+}$ coincides with $\tau_{\alpha}^{+}$which was introduced by Wade in [2].

A subset of the dyadic group is said to be a $U$-set for $\mathcal{U}_{\varepsilon}^{+}$, if $\mu \in \mathcal{U}_{\varepsilon}^{+}$and

$$
\lim _{n \rightarrow \infty} \sum_{k=0}^{2^{n}-1} \hat{\mu}(k) w_{k}(x)=0 \quad \text { except on } E
$$

imply that $\mu$ is the zero series.

THEOREM 4. A closed set of the dyadic group $E$ is a $U$-set for $U_{\varepsilon}^{+}$if and only if the $\varepsilon$-capacity of $E$ is zero.

To prove Theorem 4 we need some lemmas.

LEMMA 5. If $E$ is of $\varepsilon$-capacity zero, then

$$
\lim _{N \rightarrow \infty} \inf _{\mu \in \mathfrak{M}\left(E_{N}\right)} \sup _{x \in E_{N}}\left|U_{\mu}(x)\right|=\infty
$$

where $E_{N}=\bigcup_{x \in E} I_{N}(x)$.

PROOF. The conclusion follows from the definition of the $\varepsilon$-capacity.

LEMMA 6. If $E$ is of $\varepsilon$-capacity positive, then there exists $\mu \in \mathfrak{M}(E)$ such that $U_{\mu} \in L^{\infty}$ and $U_{\mu}(x) \geq 1 / C(E)$ a.e. on $E$.

Proof. The Walsh series of the equilibrium dyadic measure of $E$ satisfies the conclusion.

LEMMA 7. If $\mu \in U_{\varepsilon}^{+}$and $I$ is a dyadic interval, then there exist a dyadic measure $m_{\gamma}$ such that $\gamma \in \mathcal{U}_{\varepsilon}^{+}$and a positive number $n_{0}$ such that $\sum_{k=0}^{2^{n}-1} \hat{\gamma}(k) w_{k}(x)=$ $\sum_{k=0}^{2^{n}-1} \hat{\mu}(k) w_{k}(x)$ on $I$ and $=0$ otherwise for $n>n_{0}$.

PROOF. The following $m_{\gamma}$ satisfies the conclusion: $m_{\gamma}\left(I_{n}^{p}\right)=m_{\mu}\left(I_{n}^{p}\right)$ if $I_{n}^{p} \subset I$ and $=0$ otherwise.

The following lemma is Lemma 4 of [4].

LEMMA 8. If $\mu \in \mathcal{U}_{\varepsilon}^{+}$satisfies

$$
\begin{array}{cc}
\lim _{n \rightarrow \infty} \sum_{k=0}^{2^{n}-1} \hat{\mu}(k) w_{k}(x)=0 & \text { a.e. outside } F, \\
\limsup _{n \rightarrow \infty}\left|\sum_{k=0}^{2^{n}-1} \hat{\mu}(k) w_{k}(x)\right|<\infty & \text { everywhere on } F^{c} \text { except perhaps } \\
\text { on a countable set }
\end{array}
$$

when $F$ is a closed subset of the dyadic group, then for each dyadic interval $I \subset F^{c}$ there exists an integer $N$ such that $\sum_{k=0}^{2^{n}-1} \hat{\mu}(k) w_{k}(x)=0$ on I for $n \geq N$.

Proof of TheOREM 4. Assume that $E$ is not of $\varepsilon$-capacity zero. Then the equilibrium dyadic measure $m_{\mu^{*}}$ satisfies $\left\|U_{\mu^{*}}\right\|_{\infty}<\infty$. It is obvious that $\mu^{*}$ is 
not the zero series and satisfies

$$
\begin{gathered}
\lim _{n \rightarrow \infty} \sum_{k=0}^{2^{n}-1} \hat{\mu}^{*}(k) w_{k}(x)=0 \quad \text { except on } E \\
\sum_{k=0}^{2^{n}-1} \hat{\mu}^{*}(k) w_{k}(x)=2^{n} m_{\mu^{*}}\left(I_{n}(x)\right) \geq 0
\end{gathered}
$$

Since $I_{\mu^{*}} \leq\left\|U_{\mu^{*}}\right\|_{\infty}$ it is also clear that $I_{\mu^{*}}$ is finite. Hence $E$ is an $M$-set for $U_{\varepsilon}^{+}$; this contradicts the hypothesis. Then $E$ must be of $\varepsilon$-capacity zero.

To prove sufficiency let $E$ be a closed set of $\varepsilon$-capacity zero and let $\mu \in \mathcal{U}_{\varepsilon}^{+}$. By the argument of $\left[2\right.$, p. 314] it suffices to show $\hat{\mu}(0)=0$. Set $E_{n}=\bigcup_{x \in E} I_{N}(x)$ for all $N$. Since $E$ is closed, we have $E=\bigcap_{N} E_{N}$. For sufficiently large $n$,

$$
\sum_{k=0}^{2^{n}-1} \hat{\mu}(k) w_{k}(x)=0 \quad \text { except on } E_{N} .
$$

From the hypothesis $m_{\mu}$ is a nonnegative dyadic measure; then $\hat{\mu}(0) \geq 0$. From (3) we have

$$
\hat{\mu}(0)=|\hat{\mu}(0)|=\int_{E_{N}} \sum_{k=0}^{2^{n}-1} \hat{\mu}(k) w_{k}(x) d x \text { for } n>N .
$$

By Lemma 5 there exists $\mu_{N}^{*}$ such that

$$
U_{\mu_{N}^{*}}(x) \geq\left\|U_{\mu_{N}^{*}}\right\|_{\infty}=\inf _{\mu \in \mathfrak{M}\left(E_{N}\right)}\left\|U_{\mu}\right\|_{\infty} \equiv W_{\varepsilon}\left(E_{N}\right) \quad \text { on } E_{N}
$$

Therefore

$$
\begin{aligned}
|\hat{\mu}(0)| & \leq 1 / W_{\varepsilon}\left(E_{N}\right) \int_{E_{N}}\left\{\sum_{k=0}^{2^{n}-1} \hat{\mu}(k) w_{k}(x)\right\} U_{\mu_{N}^{*}}(x) d x \\
& \leq 1 / W_{\varepsilon}\left(E_{N}\right)\left\{\hat{\mu}(0)+\sum_{k=0}^{N-1} \varepsilon_{k} \sum_{\nu=2^{k}}^{2^{k+1}-1} \hat{\mu}_{N}^{*}(\nu) \hat{\mu}(\nu)\right\} .
\end{aligned}
$$

By Schwarz's inequality,

$$
\begin{aligned}
|\hat{\mu}(0)|^{2} \leq & 1 / W_{\varepsilon}\left(E_{N}\right)^{2}\left\{|\hat{\mu}(0)|^{2}+\sum_{k=0}^{\infty} \varepsilon_{k} \sum_{\nu=2^{k}}^{2^{k+1}-1}|\hat{\mu}(\nu)|^{2}\right\} \\
& \times\left\{1+\sum_{k=0}^{N-1} \varepsilon_{k} \sum_{\nu=2^{k}}^{2^{k+1}-1}\left|\hat{\mu}_{N}^{*}(\nu)\right|^{2}\right\}
\end{aligned}
$$

Since

$$
I_{\mu_{N}^{*}}=1+\sum_{n=0}^{\infty} \varepsilon_{n} \sum_{k=2^{n}}^{2^{n+1}-1}\left|\hat{\mu}_{N}^{*}(k)\right|^{2}=\left\|U_{\mu_{N}^{*}}\right\|_{\infty}=W_{\varepsilon}\left(E_{N}\right)
$$

and $\mu \in \mathcal{U}_{\varepsilon}^{+}$, we have $|\hat{\mu}(0)|^{2} \leq$ Constant $\cdot 1 / W_{\varepsilon}\left(E_{N}\right)$. By Lemma 5 and the hypothesis, we have $\lim _{N \rightarrow \infty} W_{\varepsilon}\left(E_{N}\right)=\infty$. Consequently we get $\hat{\mu}(0)=0$. 


\section{REFERENCES}

1. J. P. Kahane and R. Salem, Ensembles parfaits et séries trigonométriques, Hermann, Paris, 1963.

2. W. R. Wade, Uniqueness and $\alpha$-capacity on the group $2^{\omega}$, Trans. Amer. Math. Soc. 208 (1975), 309-315.

3. W. R. Wade and K. Yoneda, Uniqueness and quasi-measure on the group of integers of a p-series field, Proc. Amer. Math. Soc. 84 (1982), 202-206.

4. K. Yoneda, Sets of uniqueness for a certain class $\mathfrak{M}_{\varepsilon}$ on the dyadic group, Proc. Amer. Math. Soc. 89 (1983), 279-284.

Department of Mathematics, University of Osaka Prefecture, Sakai, OSAKA, JAPAN 